\title{
EVALUATION OF KNOWLEDGE, AWARENESS, AND PRACTICE OF PHARMACOVIGILANCE AMONG PRACTICING DOCTORS
}

\author{
SANTHOSH KUMAR MADHUSUDHAN*, SHASHIKALA GH
}

\author{
Department of Pharmacology, JJM Medical College, Davangere, Karnataka, India. Email: sankumar20123@gmail.com
}

Received: 18 August 2016, Revised and Accepted: 14 September 2016

\section{ABSTRACT}

Objective: The primary objective of the study was to assess the awareness, knowledge, and practice of pharmacovigilance (PV) among the practicing doctors. The secondary objective was to ascertain the reason for underreporting of adverse drug reactions (ADRs) in India.

Methods: A cross-sectional study was conducted using a pretested and validated questionnaire. The questionnaire was designed to assess these parameters of PV. These questionnaires were distributed among 90 doctors to ascertain the answers. In addition, the same parameters were reassessed (post-questionnaires) after sensitizing the doctors about PV.

Results: Only 52 doctors took the posttest, a response rate of $57 \%$. The mean awareness, knowledge, and practice of PV among doctors were 4.70 , 3.60 , and 2.90, respectively, which significantly increased in post-test questionnaire $(\mathrm{p}<0.05)$. The Pearson correlations revealed that the level of awareness among the respondents was significantly related to the knowledge and the practice of PV, linearly, and positively. In other words, higher the awareness more was the knowledge and better was the methods of application. The most common factor discouraging doctors from reporting ADRs was they found it difficult to decide whether ADR has occurred or not (32.69\%).

Conclusion: There was a significant positive correlation between awareness, knowledge, and practice of PV. In spite of these findings, the ADR reporting rate is negligible in India. To bring about a strong culture of reporting among doctors, it is mandatory to continuously expose doctors to the concepts of PV by continuous medical education, workshops, seminars and to make reporting mandatory in India.

Keywords: Pharmacovigilance, Adverse drugs reactions, Awareness, Knowledge, Practice.

(C) 2016 The Authors. Published by Innovare Academic Sciences Pvt Ltd. This is an open access article under the CC BY license (http://creativecommons. org/licenses/by/4. 0/) DOI: http://dx.doi.org/10.22159/ajpcr.2016.v9s3.14770

\section{INTRODUCTION}

Adverse drug reactions (ADRs) are an important cause of morbidity and mortality worldwide. According to the World Health Organization (WHO) definition, an ADR is any noxious, unintended, and undesired effect of a drug, which occurs at the doses which are used in humans for prophylaxis, diagnosis, or therapy [1]. Overall, the cost of drug-related morbidity and mortality exceeded $\$ 177.4$ billion in 2000, hospital admission accounted for nearly $70 \%$. This pandemic is ascribed to unnecessary prescribing, imprecise diagnosis, the outstanding development of new drugs, and unjustified promotion [2]. It has been estimated that ADR's account for approximately 5\% of all hospital admissions, $10-20 \%$ occur during hospitalization and are responsible for $7-9 \%$ of hospitalization days [3]. Even these startling figures do not represent the whole picture as these studies generally excluded ADRs caused by overdose, drug abuse, or therapeutic failures. The cost to most countries for managing ADRs is considerable and constitutes a significant economic burden on the patient and society at large. Merely, the history of years of use of a drug in millions of patients with an established drug also is not a guarantee of its safety. The recent literature of risk associated with the use of rosiglitazone (myocardial infarction) and rimonabant (psychiatric side effects) highlights the tightrope walk for physicians, who balance risk and benefits in an individual patient $[4,5]$.

Pharmacovigilance (PV) is, "The science and the activities which relate to the detection, assessment, understanding, and the prevention of adverse effects or any other drug-related problems" [6]. Even with all animal studies, clinical data, we do not know if a compound is safe for humans. At best it is "not unsafe." Thus, the physician has the most important role to play in PV, not merely because he is the first person to whom the patient will come with symptoms but also he is in a better position to suspect an ADR. The cost of a PV system, compared with the cost of ADRs to a nation and to the total national expenditure on medicines, is small. In the last decade, there has been a growing awareness that the scope of PV should be extended beyond the strict confines of detecting new signals of safety concerns. In recent past reporting of ADRs are the main cause of withdrawal of many drugs, viz., rofecoxib, cisapride, and terfenadine $[7,8]$. In a meta-analysis of 39 prospective studies from hospitals in the United States, it was shown that ADRs ranked fourth to sixth leading cause of death [9].

In a country like India, with a large population and vast diversity, it is absolutely necessary to have a standard PV program. India ranks below $1 \%$ in terms of ADR reporting against the world rate of 5\% [10]. Underreporting of ADRs is very common. It has been estimated that only $6-10 \%$ of all the ADRs are reported [11]. This high rate of underreporting is a matter of great concern which delays detection of serious ADRs, and consequently, has major negative impact on the public health. To overcome this problem, the Ministry of Health and Family Welfare, Government of India, has initiated the National PV Programme [12]

The present low level of ADR reporting is mostly due to a lack of awareness, training, and time constraints to the busy doctors. The crucial endurance is to create a culture of reporting among the health care professionals, especially among the treating physicians, as they are more closely associated with patient care. The previous reported published studies have found that underreporting of ADR is related with shortcomings in the knowledge, attitude, and perception among healthcare professionals.

Most of the similar published studies from India have not included doctors from the public health centers (PHC). These PHC doctors treat the maximum number of patients usually from a rural background with poor literacy rate; still, they are the least informed about PV. Hence, we have made a sincere attempt to include such doctors in the study. In addition, all previous studies are just passive questionnaire-based 
studies, we have gone a step ahead and sensitized the doctors with 90 minutes presentation on various aspects of PV and again captured the data post-sensitizing.

\section{METHODS}

This was a cross-sectional questionnaire-based study conducted at a tertiary care teaching hospital. The approval for conducting this study was obtained from the Institutional Ethics Committee of the College. This was an interventional study which was done among medical officers who gave their informed consent working in government PHC and doctors of various preclinical and clinical departments and postgraduates students from tertiary care teaching hospital. Those participants who did not return the questionnaires both pre- and postquestionnaire included in the stipulated time were excluded from the study. The study instrument was a pre-designed questionnaire which was structured to obtain information on the knowledge of the ADRs reporting, the attitudes toward the reporting, and the factors that in practice could hinder the reporting among the doctors. The questionnaires were set-based on similar published studies and validated. The questionnaire was designed in such a way that each question had only one correct answer. The questionnaires were administered to 90 doctors.

The questionnaire comprised 20 questions (awareness - 6 knowledge, - 5 and practice - 9). The participants were briefed about the questionnaire. They were given 20 minutes to answer the questionnaire. They could maintain anonymity with regard to their names but had to write their medical registration numbers. Once the participant doctors completed and returned the pre-questionnaire, they were sensitized to various aspects of PV in two power point presentation of 45 minutes each. Postpresentations again all doctor were given the same pre-questionnaire to evaluate their sense of understanding. The questionnaires were, then, analyzed by grading the respondents into three categories: Poor, unsatisfactory, and satisfactory as shown in Table 1.

\section{Statistical analysis}

Spearman's correlation was used to determine the relationship between awareness, knowledge, and practice of PV among the doctors. The mean and standard deviation of descriptive values was calculated, paired students t-test was used to find the statistical difference of mean. A $p<0.05$ was considered as significant. SPSS version 16 was used for analysis.

\section{RESULTS}

Out of 90 doctors, only 52 doctors took the post-test, a response rate of $57 \%$. The mean awareness, knowledge, and practice of doctors

Table 1: Grading of respondents (score range)

\begin{tabular}{lllll}
\hline Level & Poor & Unsatisfactory & Satisfactory & $\begin{array}{l}\text { Max possible } \\
\text { score }\end{array}$ \\
\hline Awareness & $1-2$ & $3-4$ & $5-6$ & 6 \\
Knowledge & $1-2$ & 3 & $4-5$ & 5 \\
Practice of PV & $1-2$ & 3 & $4-5$ & 5 \\
\hline
\end{tabular}

PV: Pharmacovigilance were $4.70,3.60$, and 2.90 , respectively, in pretest questionnaire which significantly increases in post-test questionnaire, $\mathrm{p}<0.05$, as given in Table 2.

The relationship between the awareness, knowledge, and practice of PV among the doctors was investigated using Pearson's correlation coefficient. Preliminary analyses were performed to ensure that there was no violation of the assumption of the normality and linearity. It revealed that the level of awareness among the respondents was significantly related to the knowledge and the practice of PV, linearly, and positively with correlation coefficients of 0.414 and 0.325 , with significance levels of 0.002 and 0.01 , respectively. In other words, higher the awareness more was the knowledge and better was the methods of application. Likewise, the knowledge and practice were significantly and positively related to a correlation coefficient of 0.457 , a significance level of 0.001 as shown in Table 3 .

Data were also analyzed and presented as a percent (\%) of the respondents as below.

\section{Knowledge}

Although $98 \%$ of the doctors were aware of the term PV, only $40 \%$ of doctors knew the meaning of PV. 76.92\% of doctors knew the aim of PV but $33 \%$ of doctors do not think reporting of ADRs is a professional obligation to them. The majority of doctors (88.46\%) knew that health-care professionals such as doctors, pharmacist, and nurse are responsible for reporting ADR in hospital. 92.3\% of doctors think that PV should be taught in detail to all healthcare professionals.

\section{Attitude}

Only $75 \%$ of doctors could define PV correctly, but the majority of them $(88.46 \%)$ knew the central drugs standard control organization (CDSCO) are the regulatory body responsible for monitoring ADRs. Around $45 \%$ of doctors did not know rare ADRs are detected in phase IV of clinical trials and the WHO's online database for reporting ADRs are vigiBase. Almost $80 \%$ of the doctors knew PV includes drugrelated problem, blood transfusion, herbal products, medical devices, and vaccines.

\section{Practice}

Among the participants, $73 \%$ have experienced ADRs in patients, $57.69 \%$ have seen the ADR reporting form but only $44.29 \%$ have reported an ADR to PV center. Although 84.61\% of doctors knew what constitutes serious ADR such as life-threatening event, disability, birth defects, hospitalization, and death, merely $30.76 \%$ knew that death should be reported within one day. Fortunately, the majority of doctors (88.46\%) knew ADRs are done for new drugs, major, and unexpected reactions of old drugs and any reaction or even minor reactions of new drug. Coming to the causality and severity of ADRs, only $55.76 \%$ of doctors knew it is done by Naranjo algorithm, and merely $32.69 \%$ knew severity is assessed by Modified Hartwig scale. A sincere attempt was made to find out the factors discouraging doctors from not reporting ADRs. The various reasons were no remuneration for reporting, lack of time to report ADRs due to their busy schedule, feeling a single unreported case may not affect ADR database or is not going to make a big difference. The most interesting fact was the majority of doctors, $32.69 \%$ found it was difficult to decide whether ADR has occurred or not.

Table 2: Descriptive statistics of awareness, knowledge, and practice of PV among doctors

\begin{tabular}{|c|c|c|c|c|c|c|}
\hline \multirow[t]{2}{*}{ Components } & \multicolumn{2}{|c|}{ Pre test } & \multicolumn{2}{|c|}{ Post test } & \multicolumn{2}{|c|}{ Paired t-test } \\
\hline & Mean & Standard deviation & Mean & Standard deviation & t value & $p$ value \\
\hline Awareness & 4.70 & 1.02 & 5.13 & 0.95 & -3.976 & $\mathrm{p}<0.000$ \\
\hline Knowledge & 3.60 & 1.40 & 4.34 & 1.12 & -3.825 & $\mathrm{p}<0.000$ \\
\hline Practice & 2.90 & 0.91 & 3.69 & 1.04 & -4.361 & $\mathrm{p}<0.000$ \\
\hline
\end{tabular}

All values are expressed as mean \pm SD of 52 doctors for awareness, knowledge, and practice of $P V$, means of pre-test and post-test differ significantly ( $<<0.05$ ).

PV: Pharmacovigilance, SD: Standard deviation 
Table 3: Correlation of awareness, knowledge, and method of practice of $\mathrm{PV}$ among doctors

\begin{tabular}{llll}
\hline Variable 1 & Variable 2 & $\begin{array}{l}\text { Pearson } \\
\text { correlation }\end{array}$ & p value \\
\hline Awareness & Knowledge & 0.414 & $\mathrm{p}<0.002$ \\
Awareness & Practice & 0.325 & $\mathrm{p}<0.01$ \\
Knowledge & Practice & 0.457 & $\mathrm{p}<0.001$ \\
\hline
\end{tabular}

The variables such as awareness, knowledge, and practice of PV expressed as Pearson's correlation was was linear and positively correlates significantly related $(\mathrm{p}<0.05)$. PV: Pharmacovigilance

\section{DISCUSSION}

Adverse reaction monitoring and reporting are very important in identifying the adverse reaction trends and to minimize or prevent harm to patients arising from their drugs [13]. In a country like India with multiethnic groups and a high rate of use of herbal and alternative medicine with allopathic medicines, physicians can play a major role in detecting and reporting ADRs. Various factors based on knowledge, attitude, and perception of the health professionals have been attributed for underreporting of ADRs. Inman has described them as "seven deadly sins," these includes lack of incentives, litigation, and complacency, belief that the ADR has to be reported only if it is related to the drug, indifference that this single report is not going to make a big difference, ignorance that only serious and or unexpected ADRs are necessary to be reported, and lethargy to report stating no time to report, or no forms or other excuses [14].

In the current study, only $40 \%$ knew the correct meaning of PV, 33\% of the doctors do not think reporting of ADRs are a professional obligation to them, and $45 \%$ of doctors did not know rare ADRs are detected in Phase IV of clinical trials. Similar results were seen in a study by Hardeep and Rakesh among health-care professionals in teaching hospital in northern India [15].

Among the doctors, $73 \%$ have seen ADRs in patients, $57.69 \%$ have seen the ADR reporting form but only $44.29 \%$ have reported an ADR to PV center. In a similar study done by Gupta et al. found $64.4 \%$ have experienced ADRs among patients and 58.4\% have seen an ADR reporting form, but only $22.8 \%$ have ever reported ADR to PV center [16].

The factors discouraging participants from reporting ADRs were no remuneration (5.76\%), lack of time to report ADR (50.52\%), belief that a single unreported case may not affect ADR database $(11.15 \%)$, and difficulty to decide whether ADR has occurred or not $(32.69 \%)$. In a similar study by Gupta et al. found these rates to be $31.7 \%, 23.8 \%$, $21.8 \%$, and $22.8 \%$, respectively [16]. Although this study sited lack of remuneration as the major cause of under-reporting of ADRs, our study sited difficulty to decide whether ADR has occurred or not. Whereas a study by Hardeep and Rakesh found the most common practical difficulty which was faced by the doctors in the reporting of ADRs were that a majority of them (60.6\%) did not know how and where the ADRs had to be reported.

This study revealed that the level of awareness among the responders was significantly related to knowledge and practice of PV, positively and linearly. In a similar study by Hema et al. revealed that the level of awareness among the respondents was significantly related to the knowledge and the methods of application of PV, linearly and positively with correlation coefficients of 0.346 and 0.444 , with significance levels of 0.001 and 0.001 , respectively. The knowledge and the methods were significantly and positively related with a correlation coefficient of $0.485, \mathrm{p}<0.001$ [12].

The effectiveness and success of any PV system depend highly on the knowledge, attitude, and perception of the practicing physician. To improve the reporting rate, it is vital to address the above issues in the health-care professionals with regards to the ADR reporting. There is also a requirement for constant training and enactment of regulations for ADR reporting among health-care professionals. Despite recent implementation of well-structured PV program of India in accordance with the objectives and recommendations of the WHO by CDSCO, desired success is still a distant dream [17]. With a strong knowledge of PV and using this knowledge in daily practice will enhance the number of ADRs reported. Hence, the safety data of products prescribed will increase and lead to better understanding of drugs and rational drug prescription.

\section{CONCLUSION}

This study clearly identifies gaps in awareness, knowledge, and practice of PV among doctors. There was a significant positive correlation between awareness, knowledge, and practice of PV. In spite of these findings, the ADR reporting rate is negligible in India. The major limitation of the study was the small sample size. In addition, some other factors that are associated with self-reporting studies such as accuracy of recall, personal bias could have also affected the results of this study. To bring about a strong culture of PV and reporting among doctors it is mandatory to continuously expose doctors to the concepts of PV by bringing about curriculum change in undergraduate teaching and have a regular continuous medical education, workshops, and seminars in PV or to make reporting mandatory in India.

\section{ACKNOWLEDGMENT}

We thank all doctors from JJM Medical College and medical officers from Public health centers who took part in the study. We are grateful to professor Dr. Shamshad Begum, JJM Medical College, Davangere, for carrying out the statistical analysis for this study.

\section{REFERENCES}

1. WHO. International Drug Monitoring: The Role of National Centers. Report No, 498. Geneva, Switzerland: World Health Organization; 1972.

2. Joshi SR, Sapatnekar SM. Pharmacovigilance in India: How safe are the new drugs? How safe are we? J Assoc Physicians India 2008;56:933-4.

3. Ravi V. Re-emergence of chikungunya virus in India. Indian J Med Microbiol 2006;24:83-4.

4. Kamath S, Das AK, Parikh FS. Chikungunya. J Assoc Physicians India 2006;54:725-6.

5. Robert HB Jr, Jerry RM. Muscular dystrophies and other muscle diseases. In: Harrison's, Principles of Internal Medicine. $16^{\text {th }}$ ed., Vol. II. USA: McGraw hill, Medical Publishing Division; 2005. p. 2536.

6. The Importance of Pharmacovigilance. Geneva: WHO; 2002. Available from: http://www.apps.who.int/medicinedocs/pdf/s4893e/s4893e.pdf.

7. Kumari S, Saxena A, Senthilkumar P. Evaluation of knowledge, awareness and attitude practice among nurses in pharmacovigilance at tertiary care hospital in Delhi. J Harmo Res Phar 2015;4(1):76-86.

8. Lokesh K. Pharmacovigilance/reporting adverse drug reactions: An approach to enhance health surveillance and extending market share by minimizing the chances of drug withdrawals. Int J Pharm Pharm Sci 2015;7(9):1-7.

9. Lazarou J, Pomeranz BH, Corey PN. Incidence of adverse drug reactions in hospitalised patients: A meta-analysis of prospective studies. J Am Med Assoc 1998;279:1200-5.

10. The National Pharmacovigilance Protocol, Ministry of Health and Family Welfare, Government of India. Available from: http://www. panacea-biotech.com/medicalzone/National\%20 Pharmacovigilance $\%$ 20 Protocol. pdf.

11. Feely J, Moriarty S, O'Connor P. Stimulating the reporting of an ad verse drug reaction by using a fee. Br Med J 1990;300:22-3.

12. Hema NG, Bhuvana KB, Sangeetha. Pharmacovigilance: The extent of awareness among the final year students, interns and postgraduates in a government teaching hospital. J Clin Diagn Res 2012;6(7):1248-53.

13. Sivadasan S, Sellappan M. A study on the awareness and attitude towards pharmacovigilance and adverse drug reaction reporting among nursing students in a private university, Malaysia. Int J Curr Pharm Res 2015;7(1):84-9

14. Inman WH. The attitudes to the adverse drug-reaction reporting. Br J Clin Pharmacol 1996;41:433-5. 
15. Hardeep JK, Rakesh K. A survey on the knowledge, attitude and the practice of pharmacovigilance among the health care professionals in a teaching hospital in northern India. J Clin Diagn Res 2013;7(1):97-9.

16. Gupta SK, Roopa PN, Shivaranjani R, Vidyarthi SK. A questionnaire study on the knowledge, attitude, and the practice of pharmacovigilance among the healthcare professionals in a teaching hospital in South India. Perspect Clin Res 2015;6(1):45-52.

17. Khurana A, Rastogi R, Gamperl HJ. A new era of drug safety - new eu pharmacovigilance (PV) legislation and comparison of PV in EU, US and India. Int J Pharm Pharm Sci 2014;6(7):15-21. 